\title{
Focus in Honor of Roman Zubarev, Recipient of the 2007 Biemann Medal
}

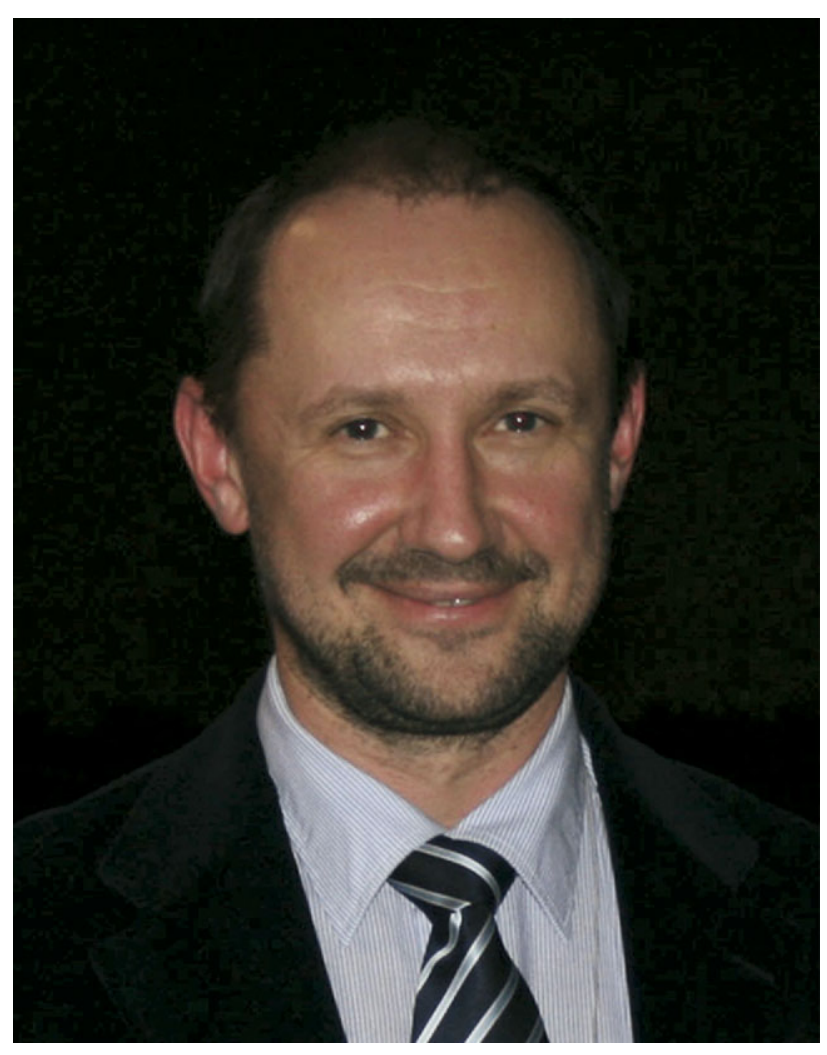

$\mathrm{T}$ The 2007 Biemann Medal was awarded to Professor Roman Zubarev at the 55th ASMS Conference for his discovery and development of electron capture dissociation (ECD) in Fourier transform ion cyclotron resonance mass spectrometry (FTICR-MS). ECD affords a new strategy for sequencing biological molecules and has already had a tremendous impact on proteomics since its initial discovery in 1998. Roman's landmark contribution has been universally recognized by the scientific community.

Roman was born in Stavropol, Russia, and remained in Russia for his early education as well as obtained a master's degree in applied physics at Moscow Engineering-Physical Institute in 1986. He earned his PhD in ion physics in 1997 at Uppsala University in Sweden, where he investigated the desorption of large biological molecules from surfaces under the direction of Bo Sundqvist. Beginning in 1997, Roman pursued postdoctoral research at Cornell University with Fred McLafferty. It was during this time that Roman discovered ECD while exploring UV photodissociation of large ions trapped in an FTICR cell. He moved to Denmark in 1998 for an appointment as an associate professor in the Department of Chemistry at the University of Southern Denmark in Odense, where he continued his studies of ion-electron reactions. In 2002, Roman became a professor at Uppsala University in Sweden, where he is currently affiliated with the Institute for Cell and Molecular Biology and Division of Molecular Biometry. His research interests in the past five years have focused on applications of ECD to proteomics and high-throughput analysis, in addition to protein network analysis. Roman expresses current fascination with "fragmentomics," which he defines as the study of the dissociation behavior of peptide ions on a large scale. His research group is also developing a "pathway search engine" that will identify activated signaling pathways from expression proteomics data.

Roman has been immensely productive and active in many aspects of science. He has published more than 120 papers in refereed journals, presented 77 lectures since 2000, and holds six patents. He has served on numerous international scientific organizations, including the advisory boards of Mass Spectrometry Reviews, European Journal of Mass Spectrometry, and Analytical Chemistry, as well as editorial boards of Rapid Communications in Mass Spectrometry, the Open Spectroscopy Journal, and Molecular and Cellular Proteomics. Roman has mentored six doctorates, two master's recipients, and ten post-doctoral fellows. Roman has also been recognized as an honorary professor at University of Southern Denmark since 2004.

This special focus issue titled "Electron Capture Dissociation" is dedicated to Roman Zubarev. This issue opens with an insight article authored by Roman that provides a critical analysis of electron capture dissociation and electron transfer dissociation in comparison to and in combination with collisional excitation methods. Roman and his co-authors conclude that the combination of two complementary fragmentation methods, one electron-based method in conjunction with collisionally activated dissociation (CAD), is essential for reliable de novo sequencing of peptides.

The focus includes contributions from six other leading laboratories that have made some of the top discoveries related to ECD and its applications. Tsybin and co-authors discuss the progress and performance of ECD in Fourier transform ion cyclotron resonance mass 
spectrometry, with an emphasis on the various electron injection techniques for implementation of ECD and a comparison of single-pass and multiple-pass ECD and the influence on ECD efficiency. A compressed hollow electron beam injection method is introduced, as well as a nonthermal electron emitter-based electron injection technique. In the third article, Evan Williams and coauthors describe the application of ion nanocalorimetry, a method in which the evaporation of water molecules from hydrated calcium clusters can be used to estimate internal energy deposition, to investigate the impact of electron kinetic energy on ECD. O'Connor and coworkers report an improvement in peptide sequence coverage obtained by ECD upon infrared heating, a result attributed to conformational changes in the peptide ions that are attributed to unfolding. The impact of charge state and sodium cationization on the electron detachment dissociation (EDD) of glycosaminoglycan oligosaccharides is discussed by the Amster group. The abundance of the $\mathrm{SO}_{3}$ loss is modulated by the charge state of the glycosaminoglycan precursor ion selected for EDD. Hakansson and co-authors report the first ECD results for acidic metabolites, namely phosphatecontaining ones, ionized by calcium complexation with electrospray ionization. In the final article in the special focus, Nielsen and colleagues discuss the electroncapture-induced dissociation of protoporphyrin cations and anions, in which cations may capture up to two electrons in successive collisions.

When Roman arrived at Cornell in early 1997 he was immediately immersed in the "strange ions" problem involving the 193-nm laser photodissociation of electrosprayed protein multiply charged ions in a Fourier transform mass spectrometer [1]. Disappointingly, these spectra mainly showed the same fragment ions as spectra from CAD and infrared multiphoton dissociation (IRMPD), but some also contained entirely different, strange fragment ions at low abundance. H. Floyd Davis, then an assistant professor at Cornell, pointed out that the 6.4-eV photons striking the FTMS ion cell walls can emit secondary electrons. Positive ions are contained on the magnetic axis of the ion cell by exterior positive electrodes; Roman and Neil Kelleher suggested a similar negative electrode reflection for any electrons produced, with Mike Senko favoring an outside electrode pair. Wonderfully, Roman's construction increased the abundance of the "strange" ions! He then "discovered" that electrons from a heated rhenium filament were even as effective as those from $\$ 30,000$ laser photons. Neil helped apply this to modified proteins and with comparisons to CAD/IRMPD—and electron capture dissociation was born [2]. In the decade since, Roman has been a leader in extending ECD and has pioneered other unique methods involving electron interactions with biomolecular cations and anions.
Even in the early years, ECD gave more than double the protein backbone cleavages that were obtained by CAD and IRMPD. The resulting huge boost of the combined techniques to "top-down" proteomics for characterization of sequence and post-translational modifications was predicted in the first ECD paper, which even suggested "top-down" as the name for this means of proteomics [2]. As of 2008, this paper has been cited 500 times in the literature and is listed by Journal of the American Chemical Society as one of its most cited articles of the last decade. Ten other publications from the Zubarev Laboratory have garnered over 100 citations to date.

Roman has established a conference on ECD and has a truly infectious passion for science and research. The ability to localize labile post-translational modifications is transforming phosphoproteomics, and Zubarev is also leading the charge to use ultra-accurate MS/MS data in proteomics research. This greatly clarifies database searching-yet another area in which Professor Zubarev has been incredibly dynamic and prolific. His contributions to such algorithms as THRASH, ModifiComb, and PhosTShunter have enabled hundreds of laboratories to capture the value of peptide fragmentation data with $<5$ part-per-million mass accuracy. This data acquisition philosophy will likely expand in the coming years, given the now widespread use of Fourier transform instrumentation-in part due to innovations directly traceable to Roman Zubarev.

ECD is already recognized as a landmark discovery and certainly has opened a new frontier of tandem mass spectrometry. The Journal of the American Society for Mass Spectrometry is honored to present this issue to showcase Roman's contribution and the impact of ECD.

Jennifer S. Brodbelt Department of Chemistry and Biochemistry University of Texas

Austin, Texas, USA

Fred W. McLafferty Department of Chemistry and Chemical Biology Cornell University Ithaca, New York, USA

Neil L. Kelleher

Department of Chemistry and the Institute of Genomic Biology University of Illinois Urbana, Illinois, USA

\section{References}

1. Guan, Z.; Kelleher, N. L.; O'Connor, P. B.; Aaserud, D. J.; Little, D. P.; McLafferty, F. W. 193 nm Photodissociation of Larger Multiply-Charged Biomolecules. Int. J. Mass Spectrom. Ion Processes 1996, 157/158, 357-364.

2. Zubarev, R. A.; Kelleher, N. L.; McLafferty, F. W. Electron Capture Dissociation of Multiply Charged Protein Cations. A Nonergodic Process. J. Am. Chem. Soc. 1998, 120, 3265-3266. 